\title{
Pairing Correlations in Odd-Mass Carbon Isotopes and Effect of Pauli Principle in Particle-Core Coupling in ${ }^{13} \mathrm{C}$ and ${ }^{11} \mathrm{Be}$
}

\author{
A.R. Samana ${ }^{1,2}$, T. Tarutina ${ }^{3}$, F. Krmpotic ${ }^{3,4,5}$, M.S. Hussein ${ }^{2}$, and T.T.S. Kuo ${ }^{6}$ \\ ${ }^{1}$ Centro Brasileiro de Pesquisas Físicas, \\ CEP 22290-180, Rio de Janeiro, RJ, Brazil \\ ${ }^{2}$ Facultad de Ciencias Exactas, Departamento de Física, \\ Universidad Nacional de La Plata, 1900 La Plata, Argentina \\ ${ }^{3}$ Departamento de Física Matemática, \\ Instituto de Física da Universidade de São Paulo, \\ Caixa Postal 66318, 05315-970 São Paulo, SP, Brazil \\ ${ }^{4}$ Instituto de Física La Plata, CONICET, 1900 La Plata, Argentina \\ ${ }^{5}$ Facultad de Ciencias Astronómicas y Geofísicas, \\ Universidad Nacional de La Plata, 1900 La Plata, Argentina and \\ ${ }^{6}$ Department of Physics and Astronomy, State University of New York, \\ Stony Brook, New York, 11794-3800, USA
}

(Dated: November 6, 2018)

\begin{abstract}
We present an exploratory study of structure of ${ }^{13} \mathrm{C},{ }^{15} \mathrm{C},{ }^{17} \mathrm{C}$ and ${ }^{19} \mathrm{C}$, showing that the simple one-quasiparticle projected BCS (PBCS) model is capable to account for several important properties of these nuclei. Next we discuss the importance of the Pauli Principle in the particle-core models of normal-parity states in ${ }^{13} \mathrm{C}$ and ${ }^{11} \mathrm{Be}$. This is done by considering the pairing interaction between nucleons moving in an over-all deformed potential. To assess the importance of pairing correlations in these light nuclei we use both the simple BCS and the PBCS approximations. We show that the Pauli Principle plays a crucial role in the parity inversion in ${ }^{11} \mathrm{Be}$. It is also found that the effect of the particle number conservation in relatively light and/or exotic nuclei is quite significant. Comparison of our results with several recent papers on the same subject, as well as with some experimental data, is presented.
\end{abstract}




\section{INTRODUCTION}

It has been known for a long time that the structure of the nucleus depends significantly on their superfluid nature. In fact, pairing constitutes the main part of the residual interaction beyond the Hartree-Fock (HF) approximation and has a strong influence on most low-energy properties of the system [1]. This encompasses masses, separation energies, deformation, individual excitation spectra and collective excitation modes such as rotations and vibrations. The role of pairing correlations is particularly emphasized when going toward the neutron drip-line because of the proximity of the Fermi surface to the single-particle continuum. Indeed, the scattering of virtual pairs into the continuum gives rise to a variety of new phenomena in ground and excited states of nuclei [2].

The above suggests that the pairing correlations might play a relevant role in the structure of light exotic nuclei. However, the fact that the ${ }^{11}$ Be ground state is a $1 / 2^{+}$state and not a $1 / 2^{-}$state (as dictates the spherical shell model) is frequently attributed to the quadrupole core excitation effects only. That is, the parity inversion in ${ }^{11} \mathrm{Be}$ is usually described within the simple particle-vibration coupling model (PVM) [3-6], or within the simple particle-rotor coupling model (PRM) [7-10], where the correlations among the valence particles, coming from the residual interaction, are totally neglected ${ }^{1}$.

We know, however, that, while the positive parity states in ${ }^{11} \mathrm{Be}$ can be accounted for fairly well in the weak coupling model [7], the low-lying negative parity states cannot be described reliably with the same model. As stated by Esbensen et al [13], this is because the last have a complicated structure due to the importance of the pairing correlations. The same statement is also valid for the ${ }^{13} \mathrm{C}$ nucleus. In fact, it has been recognized since the paper of Lane [14] on ${ }^{13} \mathrm{C}$ in the mid-50's that the weak-coupling of a $s d$ nucleon to a $p$-shell core is a good starting point for the description of low-lying non-normal-parity states in a number of $p$-shell nuclei. Moreover, Lawson and Kurath have also pointed out long ago [15] that the same is not true for the normal-parity states: "The reason is that the single-nucleon function which is added to the ${ }^{12} \mathrm{C}$ core has strong components in common

\footnotetext{
${ }^{1}$ In the early 1960's Talmi and Unna [11] first noticed the parity inversion in ${ }^{11} \mathrm{Be}$ and suggested that the interaction of a $1 p_{1 / 2}$ or $2 s_{1 / 2}$ neutron with two $1 p_{3 / 2}$ protons was the main cause. Many large-basis shell-models calculations have been carried out for ${ }^{11} \mathrm{Be}$ afterwards, but they do not all reproduce the parity inversion. Moreover, a recently done large basis ab initio shell model study [12] suggests that a realistic $3 \mathrm{~N}$ force will have an important influence on this regard.
} 
with some of the functions within the core." Thus, because of the violation of the Pauli Principle (PP) the particle-core weak-coupling models have been mostly limited to the study the positive parity spectrum of ${ }^{13} \mathrm{C}[7,15-17]$. Although important efforts have been invested to incorporate the PP into the core-particle models $[5,6,18]$, the shell-model was so far the only plausible alternative to deal with the negative parity states in odd-mass carbon and ${ }^{11}$ Be nuclei $[19,20]$.

But the pairing correlations are closely related to the PP, which is not violated within the BCS approximation, the fact that is not always well perceived. The problem that appears now, however, is the well known particle-number non-conservation. This, in turn, can be circumvented through the particle-number projection procedure [21, 22], i.e., in the framework of the projected BCS (PBCS) approximation. In fact, recently it has been found [23, 24] that the low-lying energy spectra in ${ }^{13} \mathrm{C}$ can be described quite well in the context of the PBCS approximation for the pairing force among the valence neutrons. Moreover, in this study it was shown that the projection procedure is very important to account for the weak decay observables around ${ }^{12} \mathrm{C}$.

The aim of the present work is twofold. First, we inquire to which extent the main feature of heavy carbon isotopes ${ }^{15} \mathrm{C},{ }^{17} \mathrm{C}$, and ${ }^{19} \mathrm{C}$ can be interpreted within the framework of the PBCS model, using as a building block the results obtained in Ref. [23, 24] for the stable carbon isotope ${ }^{13} \mathrm{C}$. In doing so, one should keep in mind that, as the number of neutrons increases, in going from ${ }^{13} \mathrm{C}$ to ${ }^{19} \mathrm{C}$, the nuclei become more and more weakly bound. There is no systematic study of the energy spectra of these nuclei in the literature. Secondly, we discuss the interplay between the single-particle and collective degrees of freedom in ${ }^{13} \mathrm{C}$ and ${ }^{11} \mathrm{Be}$, by incorporating the $\mathrm{PP}$ into the previous works $[3,4,7-10]$ through the pairing correlations [25-28]. Therefore, we will discuss the structure of these two nuclei in the quasiparticle-rotor model (QPRM) and the quasiparticle-vibrator model (QPVM). The corresponding models with the particle number projection included will be labelled, respectively, as PQPRM and PQPVM. Special emphasis is put on the odd-parity states which so far have been treated very rudimentary within the particle-core models.

The theoretical tools are certainly oversimplified as compared to the physical reality of nuclei that are discussed. In particular, neither the weak-binding characteristics nor coupling to the continuum states is taken into account. However, in spite of the simplicity of the approach it allows for obtaining simple estimates of the main structural features of these 
nuclei. Certainly, this kind of calculation cannot replace any full-fledged description, which is, however, presently not available. The present analysis has to be understood as a very simple version of a shell-model approach, which nevertheless is able to give main components of the involved configurations. We also feel that the paper makes some significant advance in the line of research that has been quite importantly developed by previous similar studies.

\section{PAIRING DESCRIPTION OF ODD-MASS CARBON ISOTOPES}

The definitions of particle and hole states $E_{j}^{( \pm)}$in the BCS and PBCS approximations are listed in Table I, where

$$
E_{j}=\left(\bar{e}_{j}^{2}+\Delta_{j}^{2}\right)^{1 / 2} ; \quad \bar{e}_{j}=e_{j}-\mu_{j}-\lambda,
$$

are the usual BCS quasiparticle energies, which depend on the single-particle energies (s.p.e.) $e_{j}$, on the self-energy

$$
\mu_{j}=\sum_{j^{\prime}} \frac{\left(2 j^{\prime}+1\right)^{1 / 2}}{(2 j+1)^{1 / 2}} v_{j^{\prime}}^{2} \mathrm{~F}\left(j j j^{\prime} j^{\prime} ; 0\right),
$$

and on the pairing gap

$$
\Delta_{j}=-\frac{1}{2} \sum_{j^{\prime}} \frac{\left(2 j^{\prime}+1\right)^{1 / 2}}{(2 j+1)^{1 / 2}} u_{j^{\prime}} v_{j^{\prime}} \mathrm{G}\left(j j j^{\prime} j^{\prime} ; 0\right) .
$$

TABLE I: Definitions of quasiparticle $\left(E_{j}^{(+)}\right)$, and quasihole $\left(E_{j}^{(-)}\right)$energies in the BCS and PBCS

approximations. In both cases, $E_{j}^{(+)}$can be either negative or positive, while $E_{j}^{(-)}$are always negative. The BCS quasiparticle energies $E_{j}$, defined in (2.1), are positive, and the corresponding chemical potential $\lambda$ is negative. The PBCS quasiparticle energies $\varepsilon_{j}^{N}$ are defined in (2.4).

\begin{tabular}{|c|c|c|}
\hline Model & $E_{j}^{(+)}$ & $E_{j}^{(-)}$ \\
\hline \hline BCS & $\lambda+E_{j}$ & $\lambda-E_{j}$ \\
\hline PBCS & $\varepsilon_{j}^{N}$ & $-\varepsilon_{j}^{N-2}$ \\
\hline
\end{tabular}


The PBCS energies read

$$
\varepsilon_{j}^{N}=\frac{R_{0}^{N}(j)+R_{11}^{N}(j j)}{I^{N}(j)}-\frac{R_{0}^{N}}{I^{N}} .
$$

The quantities $R^{N}$ and $I^{N}$, where $N$ is the neutron number, are defined in Ref. [21].

The BCS and PBCS predictions for the spectroscopic factors will be also discussed. Within the PBCS approximation these quantities are given by [21, 29]:

$$
S_{u}(j)=u_{j}^{2} \frac{I^{N}(j)}{I^{N}},
$$

for the stripping reactions on even targets and for pick up reactions on odd targets, and by

$$
S_{v}(j)=(2 j+1) v_{j}^{2} \frac{I^{N-2}(j)}{I^{N}},
$$

for the stripping reactions on odd targets and for pick up reactions on even targets. The plain BCS results are recovered by making all the $I$-factors equal to unity.

The BCS and PBCS calculations presented here were performed in the same way as in the previous works $[23,24]$. That is, for the residual interaction we adopted the delta force,

$$
V=-4 \pi v^{\text {pair }} \delta(r) \mathrm{MeV}-\mathrm{fm}^{3},
$$

and the configuration space includes the neutron orbital with $j \equiv n l j=$ $\left(1 s_{1 / 2}, 1 p_{3 / 2}, 1 p_{1 / 2}, 1 d_{5 / 2}, 2 s_{1 / 2}, 1 d_{3 / 2}, 1 f_{7 / 2}, 1 f_{5 / 2}\right)$. The radial wave functions were approximated by that of the harmonic oscillator $(\mathrm{HO})$ with the length parameter $b=1.67 \mathrm{fm}$, which corresponds to the estimate $\hbar \omega=\left(45 A^{-1 / 3}-25 A^{-2 / 3}\right) \mathrm{MeV}$ for the oscillator energy.

The s.p.e. $e_{j}$, as well as the value of the singlet pairing strength $v^{\text {pair }}$, were fixed by adjusting the experimental energies, taken from [32, Table 13.4], to the calculated ones, through a $\chi^{2}$ search assuming that: a) the ground state $3 / 2^{-}$in ${ }^{11} \mathrm{C}$ is the $1 p_{3 / 2}$ quasiholestate with energy $E_{1 p_{3 / 2}}^{(-)}$, and b) the lowest $1 / 2^{-}, 5 / 2^{+}, 1 / 2^{+}, 3 / 2^{+}, 7 / 2^{-}$, and $3 / 2^{-}$states in ${ }^{13} \mathrm{C}$ are the quasiparticle-states $\left.j=1 p_{1 / 2}, 1 d_{5 / 2}, 2 s_{1 / 2}, 1 d_{3 / 2}, 1 f_{7 / 2}, 2 p_{3 / 2}\right)$ with energies $E_{j}^{(+)}$. The quasiparticle energies $E_{j}^{( \pm)}$of the distant orbitals $1 s_{1 / 2}, 2 p_{1 / 2}$ and $1 f_{5 / 2}$, not known experimentally, were assumed to be: i) $E_{1 s_{1 / 2}}^{(-)} \mathrm{MeV}$ proposed in the study done by Gillet and Vinh Mau [30], ii) $E_{2 p_{1 / 2}}^{(+)}=E_{2 p_{3 / 2}}^{(+)}+\Delta_{l s}(p) \mathrm{MeV}$, with $\Delta_{l s}(p)=5.73 \mathrm{MeV}$ being the spin-orbit splitting between the $p$-subshells, and iii) $E_{1 f_{5 / 2}}^{(+)}=E_{2 p_{3 / 2}}^{(+)}+\Delta_{H O}=16.78 \mathrm{MeV}$, were $\Delta_{H O}=3.82 \mathrm{MeV}$ is the energy difference between the $1 f_{5 / 2}$ and $2 p_{3 / 2}$ states for the HO potential with standard parametrization [31]. Additionally, during the minimization 
TABLE II: The quasihole energies $E_{j}^{(-)}$with $j=1 s_{1 / 2}, 1 p_{3 / 2}$ and the quasiparticle energies $E_{j}^{(+)}$ with $j=1 p_{1 / 2}, \cdots, 1 f_{5 / 2}$, used in the fitting procedure, and the resulting single-particle energies $e_{j}$, and the pairing strength $v_{s}^{\text {pair }}$, within the BCS and PBCS for $N=6$. The energies are given in units of $\mathrm{MeV}$, and $v_{s}^{\text {pair }}$ is dimensionless.

\begin{tabular}{c|r|rc}
\hline Shell & $E_{j}^{( \pm)}$ & $e_{j}($ BCS $)$ & $e_{j}($ PBCS $)$ \\
\hline $1 s_{1 / 2}$ & -35.00 & -23.29 & -22.27 \\
$1 p_{3 / 2}$ & -18.72 & -7.74 & -7.17 \\
$1 p_{1 / 2}$ & -4.94 & -2.01 & -1.44 \\
$1 d_{5 / 2}$ & -1.09 & 2.18 & 2.22 \\
$2 s_{1 / 2}$ & -1.85 & 2.78 & 2.74 \\
$1 d_{3 / 2}$ & 3.26 & 6.88 & 6.89 \\
$1 f_{7 / 2}$ & 8.63 & 11.04 & 11.06 \\
$2 p_{3 / 2}$ & 7.24 & 11.61 & 11.62 \\
$2 p_{1 / 2}$ & 12.97 & 17.43 & 17.45 \\
$1 f_{5 / 2}$ & 16.78 & 19.27 & 19.31 \\
\hline$v_{s}^{\text {pair }}$ & & 23.46 & 24.22 \\
\hline
\end{tabular}

procedure the energy difference $e_{1 p_{3 / 2}}-e_{1 p_{1 / 2}}$ has been kept constant and equal to $\Delta_{l s}(p)$. The results are displayed in Table II.

The relationship between the s.p.e. $e_{j}$ and the corresponding quasiparticle energies $E_{j}^{(+)}$ are illustrated in Fig. 1. The major difference between the BCS and the PBCS results appears in $E_{1 s_{1 / 2}}^{(+)}$and $E_{1 p_{3 / 2}}^{(+)}$, which have not been used in the fitting procedure. Of course, opposite happens with the quasihole energies $E_{j}^{(-)}$(see Tables III and IV in Ref. [24]).

After fixing the parameterizations $\left(e_{j}\right.$ and $\left.v_{s}^{\text {pair }}\right)$ in ${ }^{13} \mathrm{C}$, we evaluate the low-lying energy spectra for the remaining odd-mass carbon isotopes, by changing only the number of neutrons. In other words, we solve the BCS and PBCS gap equations with the parameters listed in Table II and the number of particles $N=8,10$, and 12 for ${ }^{15} \mathrm{C},{ }^{17} \mathrm{C}$ and ${ }^{19} \mathrm{C}$, respectively. The results for the energy spectra and the spectroscopic factors are shown and compared with experimental data [32-43] in Figs. 2 and 3, respectively. One immediately sees that the 


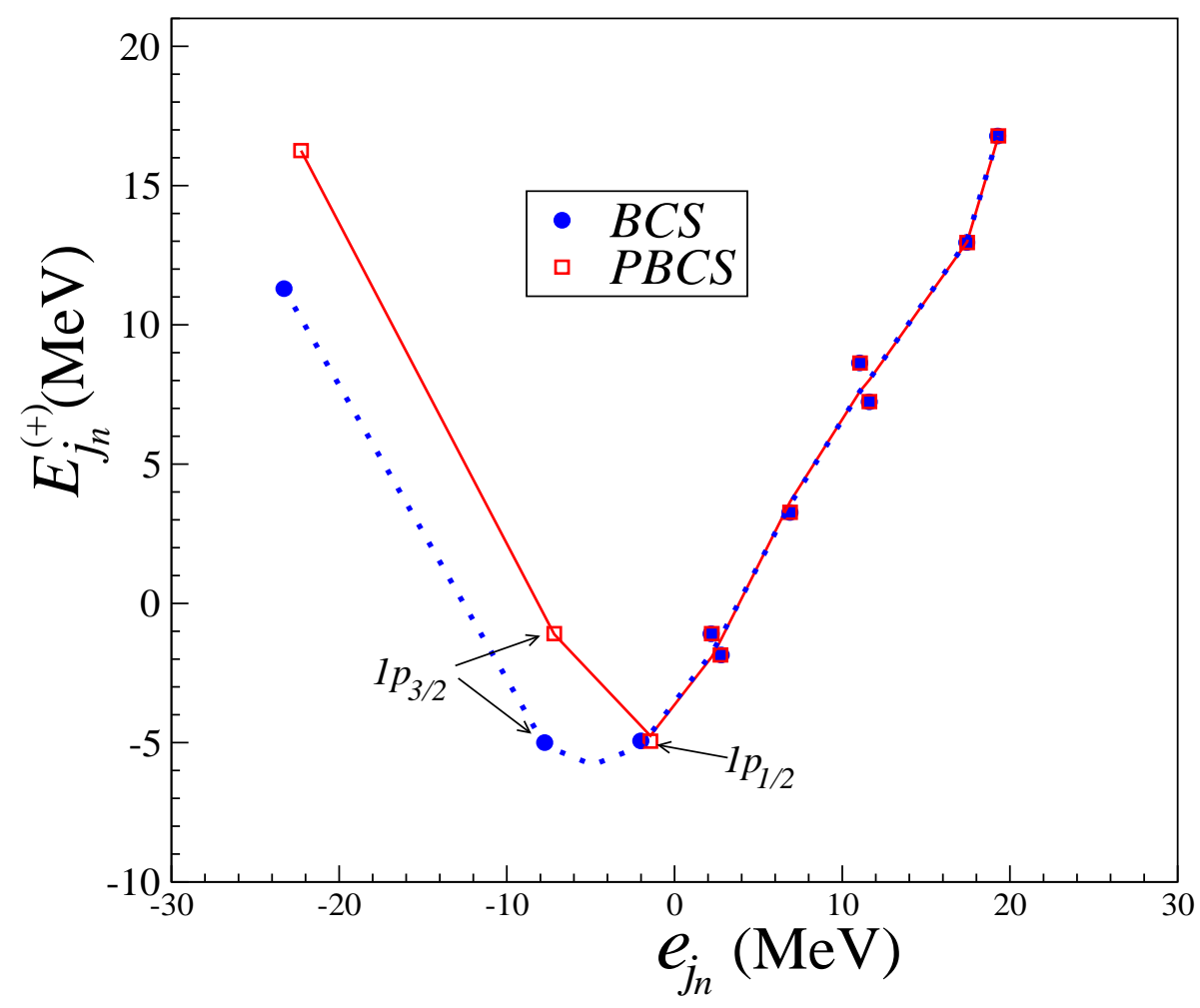

FIG. 1: Relationship between the single-particle and the quasiparticle excitation energies $E_{j}^{(+)}$for ${ }^{13} \mathrm{C}$. The states are ordered as $1 s_{1 / 2}, 1 p_{3 / 2}, 1 p_{1 / 2}, 2 s_{1 / 2}, 1 d_{3 / 2}, 1 f_{7 / 2}, 2 p_{3 / 2}, 2 p_{1 / 2}$, and $1 f_{5 / 2}$, and the energies are indicated by filled circles (BCS) and unfilled squares (PBCS).

pairing interaction reproduces to a significant extent the systematic of the energy spectra of these nuclei. In particular, the decrease in the separation energies in going from ${ }^{13} \mathrm{C}$ to ${ }^{19} \mathrm{C}$ is fairly well accounted for ${ }^{2}$.

The experimentally observed spin ordering $1 / 2^{+}, 5 / 2^{+}, 1 / 2^{-}$of the lowest three states in ${ }^{15} \mathrm{C}$, is well reproduced within the PBCS, indicating that the number projection plays a significant role, and that these levels could be predominantly one-quasiparticle (1qp) states. The present model also accounts fairly well for the $3 / 2^{-}$state at $4.66 \mathrm{MeV}$ and the $3 / 2^{+}$ state at $4.78 \mathrm{MeV}$. However, the first one of these two states could be quite likely the partner of the $5 / 2_{1}^{-}$state at $4.22 \mathrm{MeV}$, possessing therefore large, if not dominant, three-

\footnotetext{
${ }^{2}$ It would be very interesting to compare our results with a systematic large scale shell-model calculations of all four odd-mass carbon isotopes, employing the same Hamiltonian and the same single-particle space.
} 


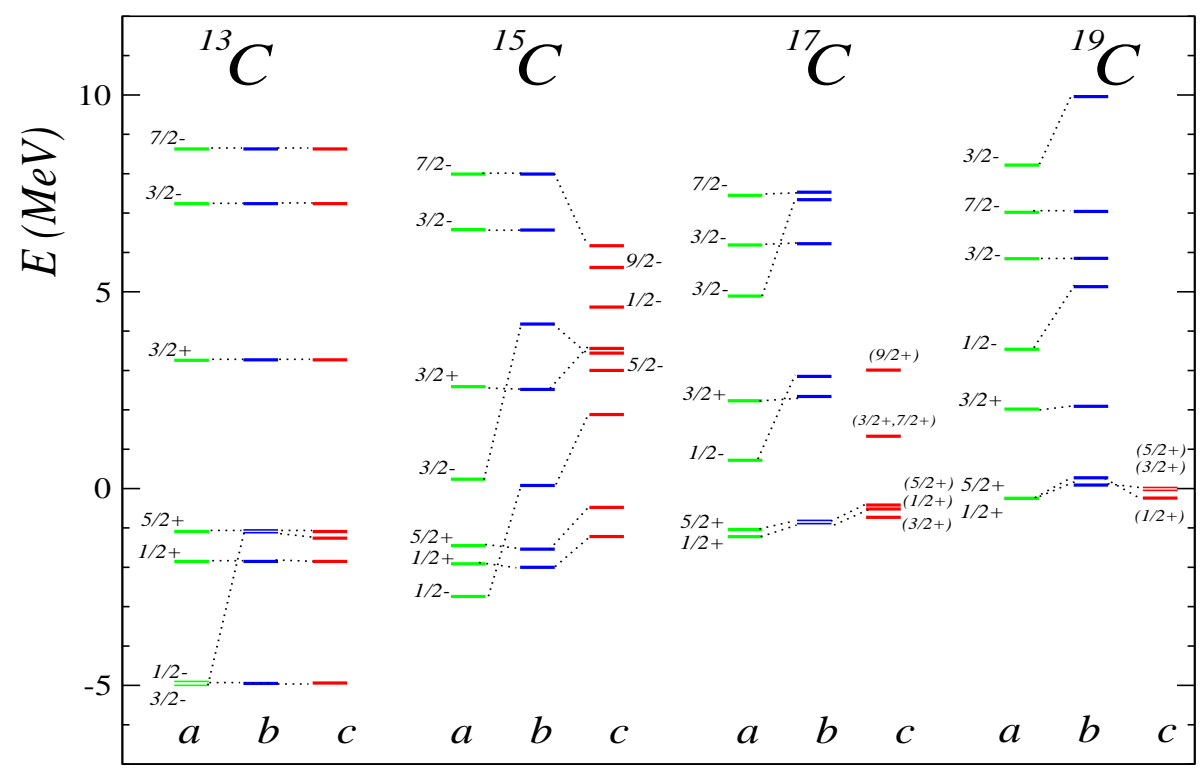

FIG. 2: Comparison between the calculated BCS, PBCS and measured level schemes for odd mass carbon isotopes: a) BCS, b) PBCS, and c) experiments: from Ref. [32] for ${ }^{13}$ C, from Refs. [3235] for ${ }^{15} \mathrm{C}$, from Refs. [36, 37] for ${ }^{17} \mathrm{C}$, and from Ref. [36] for ${ }^{19} \mathrm{C}$. For ${ }^{13} \mathrm{C}$ are only shown the experimental levels that have been used in the fitting procedure.

quasiparticle (3qp) components $\left|\left(1 d_{5 / 2}\right)^{2}, 2 ; 1 p_{1 / 2}\right\rangle$ and $\left|\left(2 s_{1 / 2}\right)^{2} ; 2,1 p_{1 / 2}\right\rangle$, as suggested by the work of Bohlen et al [34].

In ${ }^{15} \mathrm{C}$ a doublet is known from the two-neutron transfer ${ }^{13} \mathrm{C}(\mathrm{t}, \mathrm{p}){ }^{15} \mathrm{C}$ reaction [44] with possible spin assignment of $\left(9 / 2^{-}, 7 / 2^{-}\right)$, at almost the same energies $(6.84 \mathrm{MeV}, 7.39 \mathrm{MeV})$ as the $\left(3^{-}, 2^{-}\right)$doublet in ${ }^{14} \mathrm{C}$. Based on this fact, one can speculate that their $3 \mathrm{qp}$ structure is dominated by the $\left|\left(1 d_{5 / 2}\right)^{2}, 4 ; 1 p_{1 / 2}\right\rangle$ configuration [34]. The $1 / 2^{-}$level at $5.87 \mathrm{MeV}$ could be a 3qp (seniority one or three) state which is not contained in our configuration space. In fact, our restriction to 1qp subspace eliminates many of the well known states in ${ }^{15} \mathrm{C}$. However, except for the $5 / 2_{1}^{-}$state, the energy spectrum compares fairly well with the experimental results and with a recent shell-model calculation (cf. Fig. 2 in Ref. [20]). 
The inversion of the $5 / 2_{1}^{+}$and $1 / 2_{1}^{+}$states in ${ }^{15} \mathrm{C}$, regarding the standard single-particle ordering of levels, which occurs in ${ }^{17} \mathrm{O}$, has been discussed a long time ago by Talmi and Unna [11]. They have shown, in a brilliant manner, that the crossing of these two levels, in going from ${ }^{17} \mathrm{O}$ to ${ }^{15} \mathrm{C}$, comes from difference in interaction energy of the $1 d_{5 / 2}$ and $2 s_{1 / 2}$ neutrons with two removed $1 p_{1 / 2}$ protons ${ }^{3}$. Nevertheless, as this phenomenon is typical of weakly-bound light neutron rich nuclei, it is seldom considered to be an "exotic" feature of ${ }^{15} \mathrm{C}[35,45]$. That is, it is attributed to the halo formation, which makes the lowest angular momentum to gain energy by extending its wave function. This apparent "anomaly" even takes place in the non-exotic ${ }^{13} \mathrm{C}$ nucleus, and in our model it can be interpreted as a consequence of the pairing interaction. In fact, as seen from Table II and Fig. 2, we obtain that for all odd carbon isotopes is $E_{1 d_{5 / 2}}^{(+)}>E_{2 s_{1 / 2}}^{(+)}$, although $e_{1 d_{5 / 2}}<e_{2 s_{1 / 2}}$. This is a direct consequence of fact that the $2 s_{1 / 2}$ self-energy is significantly larger than that of the $1 d_{5 / 2}$ state, because of the strong interaction between the $1 s_{1 / 2}$ and $2 s_{1 / 2}$ states (see Eq. (2.2)). It might be worthwhile to point out that the effective s.p.e. $e_{j}$ used here should not be confounded with the ${ }^{17} \mathrm{O}$ energy spectrum.

The separation energy of the last neutron in ${ }^{17} \mathrm{C}$ is $S_{n}=729 \pm 18 \mathrm{keV}$ [46], and the shell model calculation [47] predicts a $J^{\pi}=3 / 2^{+}$ground state. This prediction has been confirmed later on by the single-neutron knockout reaction measurements done by Maddalena et al [39], which strongly indicates such an assignment instead of the naively expected option $J^{\pi}=5 / 2^{+}$arising from the seniority-one state $\left|\left(1 d_{5 / 2}\right)^{3} J^{\pi}=5 / 2^{+}\right\rangle$. A simple explanation for this experimental result could be found in the so called $J=j-1$ anomaly discussed by Bohr and Mottelson [48]. In fact, since the work of Kurath [49] we know that for $(j)^{3}$ configurations with $j \geq 5 / 2$, the $J=j-1$ state can occur below the $J=j$ state for sufficiently long range forces ${ }^{4}$. The model discussed here does not contain seniority-three states and therefore it is unable to account for the $3 / 2^{+}$ground state spin. However, as seen from the Fig. 2, it predicts that the first two excited states are $1 / 2^{+}$and $5 / 2^{+}$(in this order), which

\footnotetext{
${ }^{3}$ The fact that ${ }^{17} \mathrm{C}$ has a low-lying $1 / 2_{1}^{+}$state, and ${ }^{19} \mathrm{C}$ has a $1 / 2_{1}^{+}$ground state could also be a consequence of the same $1 p_{1 / 2}^{-1} 1 d_{5 / 2}$ and $1 p_{1 / 2}^{-1} 2 s_{1 / 2}(T=1)$ particle-hole matrix elements, lowering configurations involving the $1 / 2_{1}^{+}$states in ${ }^{19} \mathrm{O}$ and ${ }^{21} \mathrm{O}$ at $1.47 \mathrm{MeV}$ and $1.22 \mathrm{MeV}$, respectively, very much in the same way as for ${ }^{15} \mathrm{C}$.

${ }^{4}$ The $3 / 2^{+}$ground state in ${ }^{17} \mathrm{C}$ can similarly be attributed to the differential effect of above mentioned particle-hole interaction on the $2 s_{1 / 2}$ content of the dominantly $\left(1 d_{5 / 2}\right)^{3} 5 / 2_{1}^{+}$and $3 / 2_{1}^{+}$states of ${ }^{19} \mathrm{O}$.
} 


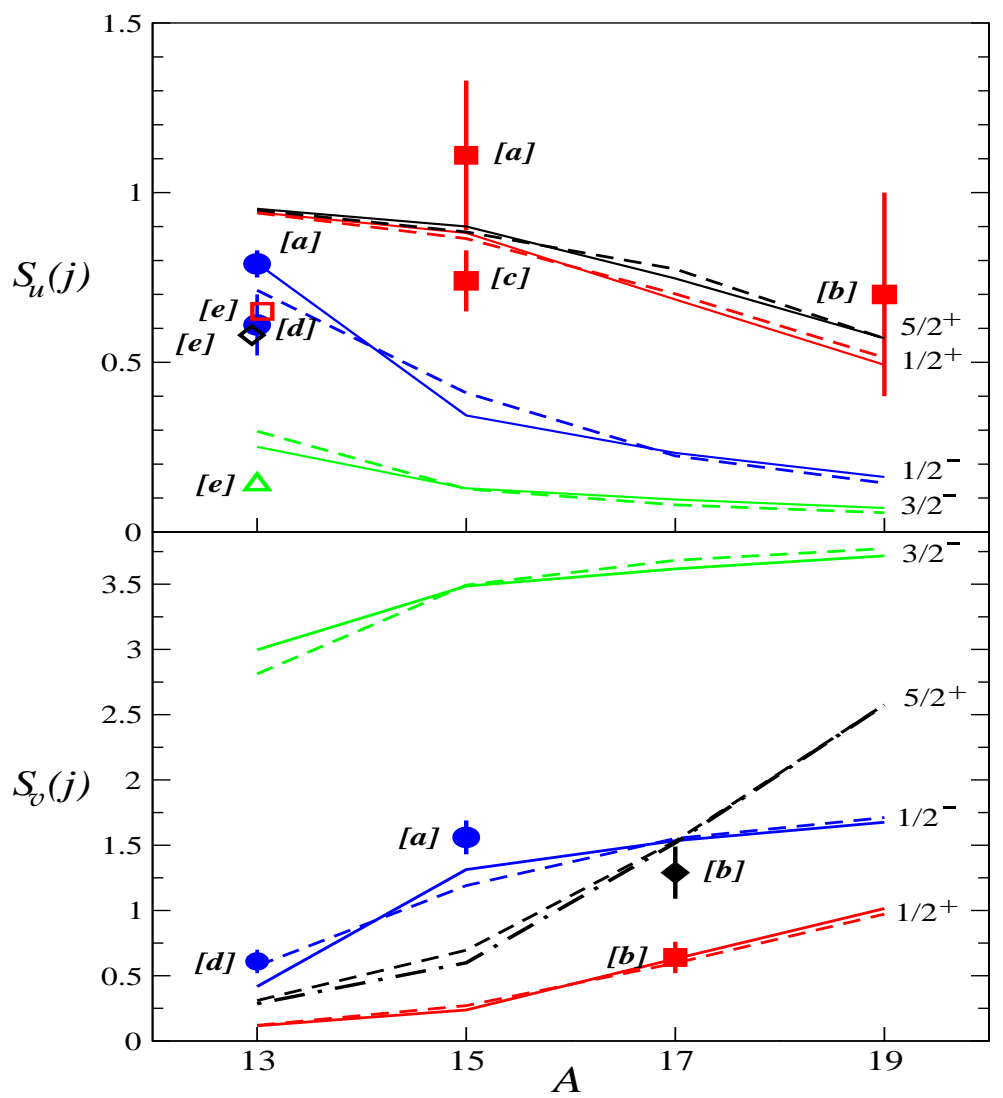

FIG. 3: (Color online) One-particle reaction spectroscopic factors as a function of the mass number $A$. Upper panel: $S_{u}(j)$, for stripping on even parent and pick-up/ knock-out on odd parent. Lower panel: $S_{v}(j)$, for pick-up/knock-out on even parent and stripping on odd parent. The dashed and solid lines correspond, respectively, to the BCS and PBCS predictions. The experimental values for the lowest $J^{\pi}=1 / 2^{-}, 3 / 2^{-}, 1 / 2^{+}$and $5 / 2^{+}$states are indicated, respectively, with circles, triangles, squares, and diamonds. The data were by recompiled from Refs.: $[38]^{[a]},[39,40]^{[b]},[40,41]^{[c]},[42]^{[d]}$ and $[43]^{[e]}$.

is consistent with the recent measurement done by Elekes et al [36].

Special attention was given to the neutron-rich carbon isotope ${ }^{19} \mathrm{C}$ to establish whether it has a pure $s_{1 / 2}$ one-neutron-halo as suggested in Refs. [10, 50]. On the basis of measurements of different observables, associated with the neutron-removal reaction [39, 51, 52], there seems to be the consensus that the spin and parity of its ground state is $J^{\pi}=1 / 2^{+}$. Contrarily, there is a strong discrepancy in the literature regarding the separation energy of 
${ }^{19} \mathrm{C}$. The tabulated values go from $S_{n}=160 \pm 95 \mathrm{keV}$ in $1993-1997$ [53] to $S_{n}=580 \pm 90$ $\mathrm{keV}$ in 2003 [54]. The experiments using time-of-flight techniques suggest small separation energy, that is, weighted average yields $S_{n}=242 \pm 95 \mathrm{keV}$ [50]. The Coulomb dissociation of ${ }^{19} \mathrm{C}$ was studied by Nakamura in [51], and the analysis of angular distributions of breakup products suggests the value $S_{n}=0.53 \pm 0.13 \mathrm{MeV}$. Using this value in the simple cluster model calculation of the dipole strength gives good agreement with the data. On the other hand, more recent experiment of Maddalena et al. [39] on nuclear breakup of ${ }^{19} \mathrm{C}$ yields $S_{n}=0.65 \pm 0.15 \mathrm{MeV}$ and $0.8 \pm 0.3 \mathrm{MeV}$. In the present calculation we correctly reproduce the spin and parity of the ground state. For its energy we obtain $-0.33 \mathrm{MeV}$ in the BCS and $0.12 \mathrm{MeV}$ in the PBCS. Both results are in fair agreement with the values reported in Refs. [50,53]. Two excited states at energies of $197(6) \mathrm{keV}$ and $269(8) \mathrm{keV}$ were reported in a recent study of the $\gamma$-ray spectra [36]. The suggested spins and parities are $3 / 2^{+}$ and $5 / 2^{+}$, and, as seen from Fig. 2, we reproduce the second one only. The level $3 / 2^{+}$is very likely dominated by the seniority-three configuration $\left|\left(1 d_{5 / 2}\right)^{3} J^{\pi}=3 / 2^{+}\right\rangle$, same as the ground state in ${ }^{17} \mathrm{C}$.

The BCS and PBCS one-particle reaction spectroscopic factors for the lowest $J^{\pi}=$ $1 / 2^{-}, 3 / 2^{-}, 1 / 2^{+}$and $5 / 2^{+}$states in odd-mass carbon isotopes are shown in Fig. 3 , as a function of the mass number $A$. They are quite similar to each other, and, except for ${ }^{13} \mathrm{C}$, they also agree fairly well with the experimental data which are displayed in the same figure. This agreement clearly implies that in ${ }^{15} \mathrm{C}-{ }^{19} \mathrm{C}$ nuclei the states $J^{\pi}=1 / 2^{-}, 1 / 2^{+}$and $5 / 2^{+}$ are basically seniority-one states. In contrast, the contribution of seniority-three states, and/or of the collective degrees of freedom seems to be quite relevant in ${ }^{13} \mathrm{C}$. The latter will be discussed in the next section.

\section{III. ${ }^{13} \mathrm{C}$ AND ${ }^{11} \mathrm{BE}$ WITHIN THE QUASIPARTICLE-CORE MODEL}

The quasiparticle-rotor model hamiltonian is evaluated in the same way as in the PRM [710], except for:

1. The band-head energies are modified as:

$$
e_{j} \rightarrow E_{j}^{(+)}
$$


with $E_{j}^{(+)}$defined in Table I, and

2. The non-diagonal particle-core matrix elements are renormalised by the overlap factors:

$$
F_{j j^{\prime}}^{Q P}=u_{j} u_{j^{\prime}}-v_{j} v_{j^{\prime}}
$$

in the QPRM, and

$$
F_{j j^{\prime}}^{P Q P}=\frac{u_{j} u_{j^{\prime}} I^{N}\left(j j^{\prime}\right)-v_{j} v_{j^{\prime}} I^{N-2}\left(j j^{\prime}\right)}{\left[I^{N}(j) I^{N}\left(j^{\prime}\right)\right]^{1 / 2}},
$$

in the PQPRM.

TABLE III: Neutron Wood-Saxon s.p.e. $e_{j}$ for ${ }^{13} \mathrm{C}$ and ${ }^{11} \mathrm{Be}$ and the corresponding quasiparticle BCS and PBCS energies $E_{j}^{(+)}$, together the energies $E\left(2^{+}\right)$of the collective $2^{+}$states. All energies are given in units of $\mathrm{MeV}$, and the pairing strength is $v_{s}^{\text {pair }}=30$.

\begin{tabular}{l|rrr|rrr}
\hline \hline Shell & \multicolumn{3}{|c|}{${ }^{13} \mathrm{C}$} & \multicolumn{3}{c}{${ }^{11} \mathrm{Be}$} \\
& $e_{j}$ & \multicolumn{2}{|c|}{$E_{j}^{(+)}$} & $e_{j}$ & \multicolumn{3}{c}{$E_{j}^{(+)}$} \\
& & BCS & PBCS & & BCS & PBCS \\
\hline $1 p_{3 / 2}$ & -13.54 & -9.5956 & -7.109 & -7.33 & -3.5928 & -1.8592 \\
$1 p_{1 / 2}$ & -7.82 & -10.4057 & -12.209 & -2.59 & -3.9583 & -5.4577 \\
$1 d_{5 / 2}$ & -0.82 & -4.2699 & -4.123 & 3.50 & 0.2640 & 0.2333 \\
$2 s_{1 / 2}$ & -0.61 & -3.4033 & -3.303 & 0.30 & -2.0479 & -2.1316 \\
\hline$E\left(2^{+}\right)$ & & & 4.438 & & & 3.368 \\
\hline
\end{tabular}

Thus, the PRM differs in several important aspects from the QPRM and PQPRM. First, as shown in Fig. 1, the BCS and PBCS energies $E_{j}^{(+)}$can be quite different from the s.p.e. $e_{j}$, not only in magnitude but also in sign. Second, the factors $F_{j j^{\prime}}$, which are quite similar in BCS and PBCS, correctly take in account the PP and they can by considerable less than unity for states near the Fermi level, diminishing in this way their coupling to the core excited states quite a lot. In addition, the particle-like states do not couple to the hole-like states, and if the particle-core coupling is attractive (repulsive) for a particle-like state $j$, it is repulsive (attractive) for a hole-like state with same quantum numbers $j$.

The vibrational analog of the QPRM, i.e., the quasiparticle-vibrator coupling model (QPVM) has been introduced in Refs. [27, 28, 48]. Note also that when only the collective 


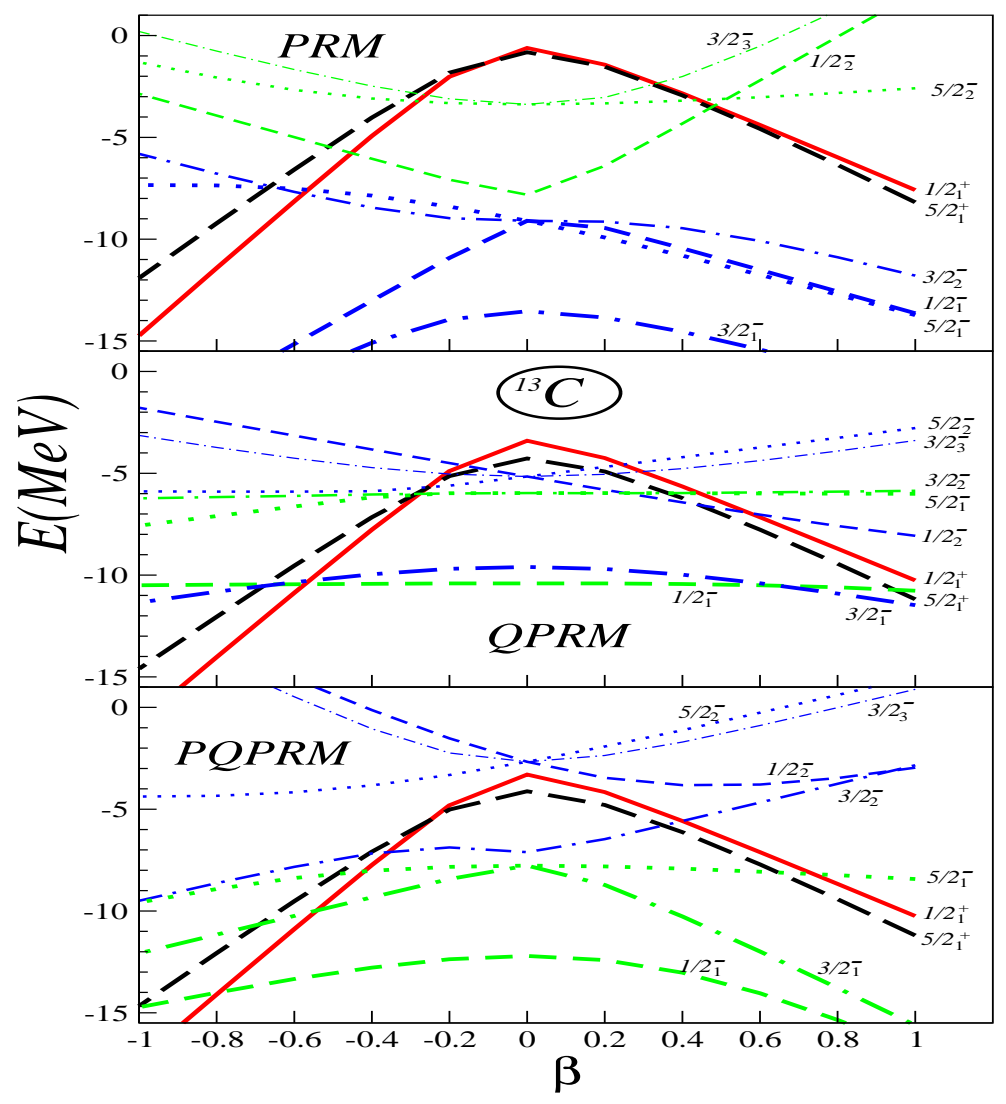

FIG. 4: (Color online) Calculated levels for ${ }^{13} \mathrm{C}$ as functions of the $\beta$ value for ${ }^{12} \mathrm{C}$, within the PRM, QPRM and PQPRM models.

state $2^{+}$is considered, and the diagonal $2^{+}-2^{+}$interactions are neglected, the QPRM and QPVM formally yield the same result, except that the negative value of the deformation parameter $\beta$ does not have any physical meaning in the vibrational case. The same statement is valid for the projected version of the QPVM, i.e., for the PQPVM.

Incidentally the s.p.e., used in the previous section and shown in Table II, were extracted from the experimental data and thus already include the collective degrees of freedom. As such, they can not be used in the core-particle models. Moreover, due to the fact that the results strongly depend on the size of the configuration space, and in order to make the comparison with previous particle-core calculations $[3,4,7-10]$ as close as possible, we will use here only four bare s.p.e., obtained from a Wood-Saxon potential with standard parametrization [55]. The results for ${ }^{13} \mathrm{C}$ and ${ }^{11} \mathrm{Be}$ are shown in Table III. Being the single- 


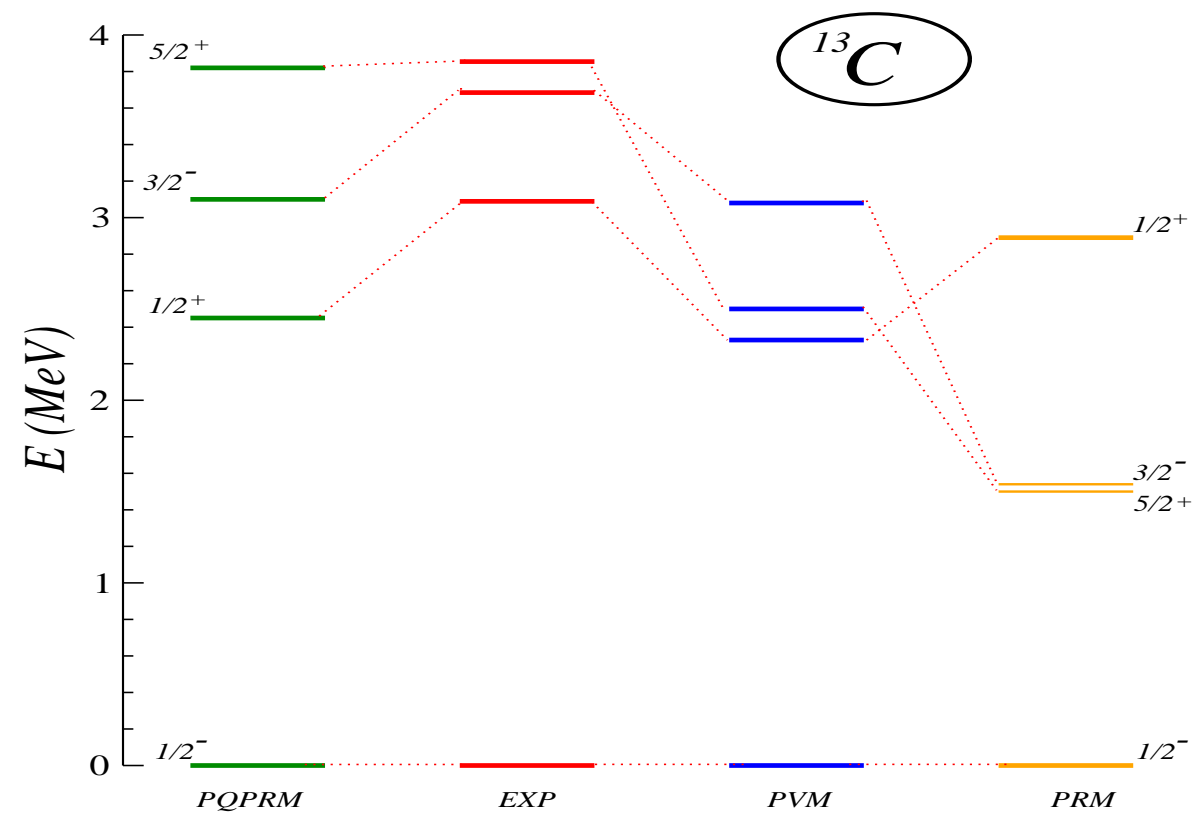

FIG. 5: (Color online) The low-energy spectra in ${ }^{13} \mathrm{C}$ within the PQPRM for $\beta=-0.6$, compared with the experimental levels (EXP) and the PVM [3] and PRM [9] calculations.

particle space here smaller than the one used in the previous section, we employ now a somewhat larger pairing strength, in order to achieve the convergence of the BCS equations. We wish to stress that all the discussion done within the particle-core models is basically qualitative, and therefore the choice of the model parameters (Wood-Saxon potential, $E\left(2^{+}\right.$), $v_{s}^{\text {pair }}$, and $\beta$ ) doesn't play a crucial role here.

The calculated low-lying levels for ${ }^{13} \mathrm{C}$ as functions of the $\beta$ value for ${ }^{12} \mathrm{C}$, within the PRM, QPRM and PQPRM models are shown in Fig. 4. Let us first note that the lowest states $J^{\pi}=3 / 2_{1}^{-}, 1 / 2_{1}^{-}, 3 / 2_{2}^{-}$and $5 / 2_{2}^{-}$, based on the unperturbed configurations $\left|1 p_{3 / 2}, 0^{+}\right\rangle$ and $\left|1 p_{3 / 2}, 2^{+}\right\rangle$, are frequently just ignored within the particle-core coupling models $[3,4$, $8,9]$ in order to simulate the $\mathrm{PP}^{5}$. We are considering them, however, in order to make the comparison with the QPRM and PQPRM models, where these states are physically meaningful. Moreover, the state $J^{\pi}=1 / 2_{2}^{-}$, build up on $\left|1 p_{1 / 2}, 0^{+}\right\rangle$, is considered to be the

\footnotetext{
${ }^{5}$ In Ref. [7] have not been considered the negative parity states.
} 
ground state in the PRM.

The measured quadrupole moment of the core ${ }^{12} \mathrm{C}$ is $Q_{0}=-(22 \pm 10)$ e $\mathrm{fm}^{2}$ [56], suggesting an oblate shape. This gives a quite large quadrupole deformation $(\beta \cong-0.6)$. As can be seen from Fig. 4, only the PQPRM reproduces satisfactorily the experimental energy ordering of the lowest four levels in ${ }^{13} \mathrm{C}$ with this value of $\beta$. In Fig. 5 we confront the PQPRM energies for this $\beta$ of the lowest $1 / 2^{-}, 3 / 2^{-}, 1 / 2^{+}$and $5 / 2^{+}$levels in ${ }^{13} \mathrm{C}$ with the experimental data, and the PVM [3] and PRM [9] calculations.

It should be noted that the PRM and QPRM are unable to account for the ${ }^{13} \mathrm{C}$ energy spectra for any value of the deformation parameter, neither positive nor negative. In the first case, and even when the levels based on the configurations $\left|1 p_{3 / 2}, 0^{+}\right\rangle$and $\left|1 p_{3 / 2}, 2^{+}\right\rangle$ are omitted, a low lying $5 / 2^{-}$state, arising from the unperturbed $\left|1 p_{1 / 2}, 2^{+}\right\rangle$level, shows up, which is not observed experimentally. On the other hand, in the second case, the particlecore coupling never removes the degeneracy between the states $1 / 2_{1}^{-}$and $3 / 2_{1}^{-}$.

The resulting PRM, QPRM and PQPRM wave functions for the lowest $1 / 2^{-}, 3 / 2^{-}$, $1 / 2^{+}$, and $5 / 2^{+}$states, with $\beta=-0.6$, are confronted with previous calculations $[3,7,9]$ in Table IV. As mentioned before, within the PRM are ignored the states $J^{\pi}=3 / 2_{1}^{-}, 1 / 2_{1}^{-}$, and $3 / 2_{2}^{-}$, based on the unperturbed configurations $\left|1 p_{3 / 2}, 0^{+}\right\rangle$and $\left|1 p_{3 / 2}, 2^{+}\right\rangle$, and are exhibited the wave functions of the $1 / 2_{2}^{-}$and $3 / 2_{3}^{-}$levels. On the other hand, in the PRM calculations done in Refs $[7,9]$ instead of the wave functions are presented the percentages of the different configurations, and therefore we only show absolute values of the corresponding amplitudes.

It is worth noting that, while the pairing makes the wave functions of the negative parity states less collective, all three models used here yield similar results for the positive parity states. We find that about $55 \%$ of the $1 / 2_{1}^{+}$state consists of the $1 d_{5 / 2}$ single-particle state coupled to the $2^{+}$excited core state. In contrast, in the previous particle-core coupling calculations $[3,7,9]$ this state is basically $(\geq 90 \%)$ of single-particle nature. As seen from the Table IV, our wave functions for the $5 / 2_{1}^{+}$are also significantly more collective that those obtained in the just mentioned works.

The differences in the wave functions, displayed in the Table IV, are reflected in the reaction spectroscopic factors for pickup on the ${ }^{13} \mathrm{C}$ target and for stripping on the ${ }^{12} \mathrm{C}$ target, which are shown in the same table. Also are listed the results of the previous PVM and PRM [3, 7, 9], and shell model [19] studies, as well as the experimental results [42, 43, 57], which are accounted for quite well within the present PQPRM calculations. 
TABLE IV: Comparison between the wave functions and the spectroscopic factors obtained in the present work for the lowest states in ${ }^{13} \mathrm{C}$ with those derived by Vinh Mau [3] within the PVM, and by Nunes et al [9] and Esbensen et al [7] within the PRM, and the shell model study done by Cohen and Kurath [19]. In all our calculations (PRM, QPRM, and PQPRM) we adopt $\beta=-0.60$.

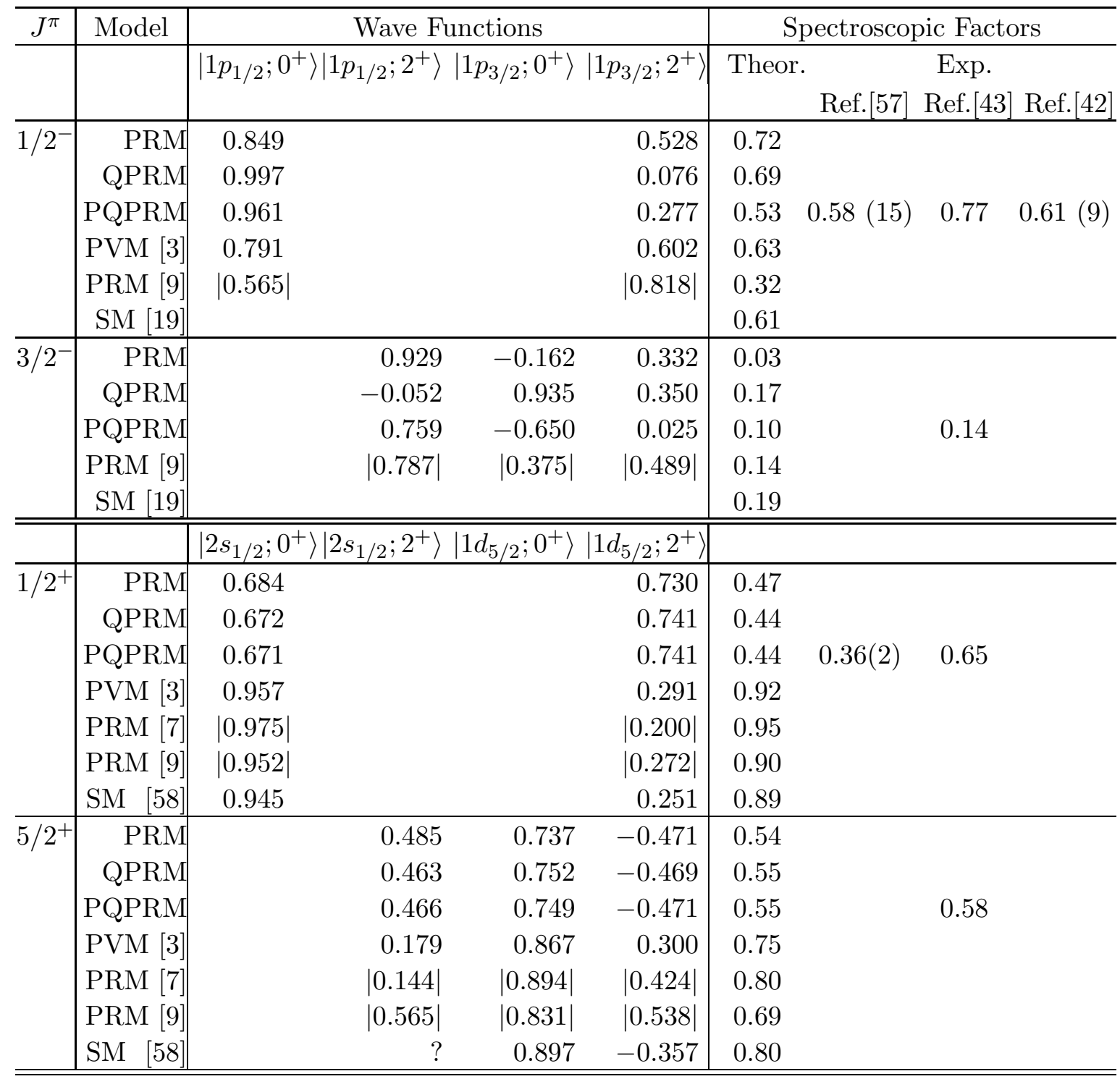

We obtain much smaller amplitudes for the non-collective components $\left|2 s_{1 / 2} ; 0^{+}\right\rangle$and $\left|1 d_{5 / 2} ; 0^{+}\right\rangle$of the lowest $\left|1 / 2_{1}^{+}\right\rangle$and $\left|5 / 2_{1}^{+}\right\rangle$states than do the full $1 \hbar \omega$ shell-model calculations, among which the work of Jager et al [59] was very likely the first one. There have been many such calculations since then with refined effective interactions, but the wave functions for the lowest positive-parity states remain essentially unchanged. We show the 
ones obtained in the work done by Millener et al [58], which, as seen from Table IV, yield spectroscopic factors that are $\sim 30-60 \%$ larger than the measured ones.

In Fig. 6 we show the PRM, QPRM and PQPRM results of our study of the low-lying states in ${ }^{11} \mathrm{Be}$ as a function of deformation. All what has been pointed out in commenting the ${ }^{13} \mathrm{C}$ nucleus in the PRM, regarding the PP, the unperturbed configurations $\left|1 p_{3 / 2}, 0^{+}\right\rangle$ and $\left|1 p_{3 / 2}, 2^{+}\right\rangle$, and the negative parity states, is also pertinent here. On the other hand, when comparing our PRM results with the work of Esbensen et al [7] one sees that their deformation dependence of the levels $1 / 2^{+}$and $5 / 2^{+}$gets flatter than ours for strong deformations. The reason for that is simple and comes from the fact that they use a volume factor in the calculation, given by $[7,(3)]$, to preserve the volume of the nucleus. This actually means that their interaction radius is smaller for higher deformations and this results in the flattening of the curves. Having said that, it should be noted that our PRM curves for the states $1 / 2_{1}^{+}$and $1 / 2_{2}^{-}$are very much like those in [9, Fig. 2]. The main difference is that in our case the crossings between the positive and negative $1 / 2$ states occur at a significantly smaller value of $\beta$.

Both PRM calculations performed so far $[7,9]$ were done with a positive value of $\beta$, i.e., a prolate deformation has been assumed for the ${ }^{10} \mathrm{Be}$ nucleus. However, there is no firm experimental evidence that it is so. Furthermore, we neither know whether ${ }^{10}$ Be behaves as a rotator or as a vibrator. From its energy spectra, with the first and second $2^{+}$at energies $E_{2_{1}^{+}}=3.37 \mathrm{MeV}$ and $E_{2_{2}^{+}}=5.96 \mathrm{MeV}$ (i.e., $E_{2_{2}^{+}} \cong 2 E_{2_{1}^{+}}$), one can conclude that it is more likely a vibrator. In fact, Esbensen et al [7] have noted that for the positive parity spectra in ${ }^{11} \mathrm{Be}$ a better agreement with data is obtained after reducing (in 34\%) the $2^{+}-2^{+}$coupling strength. On the other hand, Vinh Mau [3] and Coló et al [4] use straightforwardly the PVM.

The most recent study of quadrupole deformation of ${ }^{10} \mathrm{Be}$ has been done through proton inelastic scattering [60], and the values for $\beta$, extracted from the measured deformation lengths $\delta=\beta R=\beta r_{0} A^{1 / 3}$, are: $\beta=0.593(56)$ and $\beta=0.692(65)$. A careful scrutiny of the energy levels in Fig. 6, leads one to conclude that none of our particle-rotor coupling calculations is able to reproduce the experimentally observed spin sequence $1 / 2^{+}-1 / 2^{-}-$ $5 / 2^{+}$in ${ }^{11} \mathrm{Be}$, for such a value of $\beta$ neither positive nor negative. In view of this it does not make much sense to comment the wave functions within the PRM, QPRM and PQPRM approximations. 


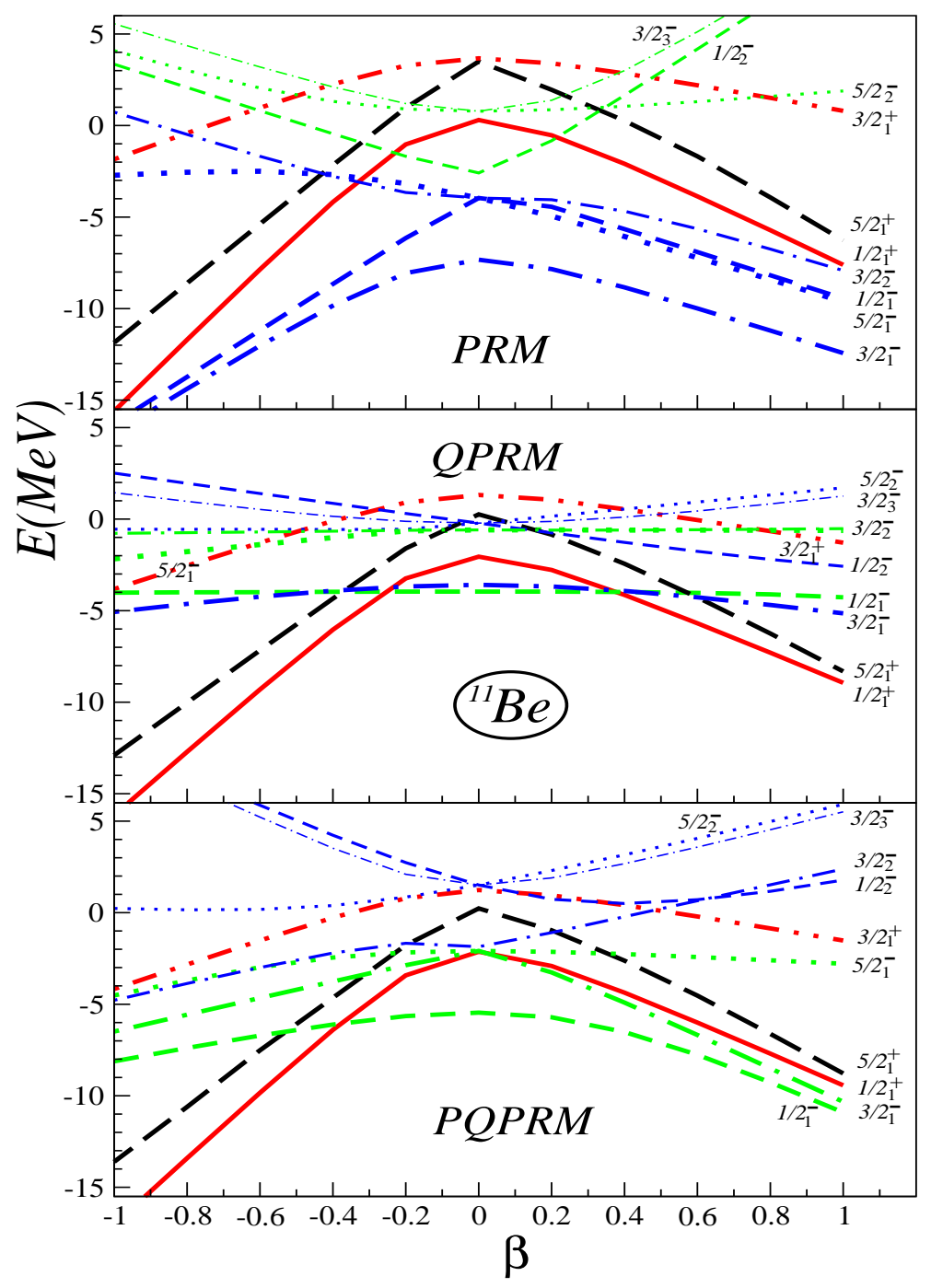

FIG. 6: (Color online) Calculated levels for ${ }^{11} \mathrm{Be}$ as functions of the $\beta$ value for ${ }^{10} \mathrm{Be}$, within the PRM, QPRM and PQPRM models.

The just mentioned results have induced us to replace the rotor by a harmonic vibrator in the description of the low-lying ${ }^{11}$ Be levels. The results are shown in Fig. 7. The PVM (upper panel) exhibits the same features as the PRM regarding the PP. Therefore the lowest four negative parity states, same as in the PRM, have to be discarded within the PVM. However, even doing so it is not possible to obtain the desired theoretical results. On the other hand, from the QPVM energy spectra (middle panel) one sees that the particle-vibrator coupling, same as the particle-rotor coupling, is unable to remove the degeneracy between the states 


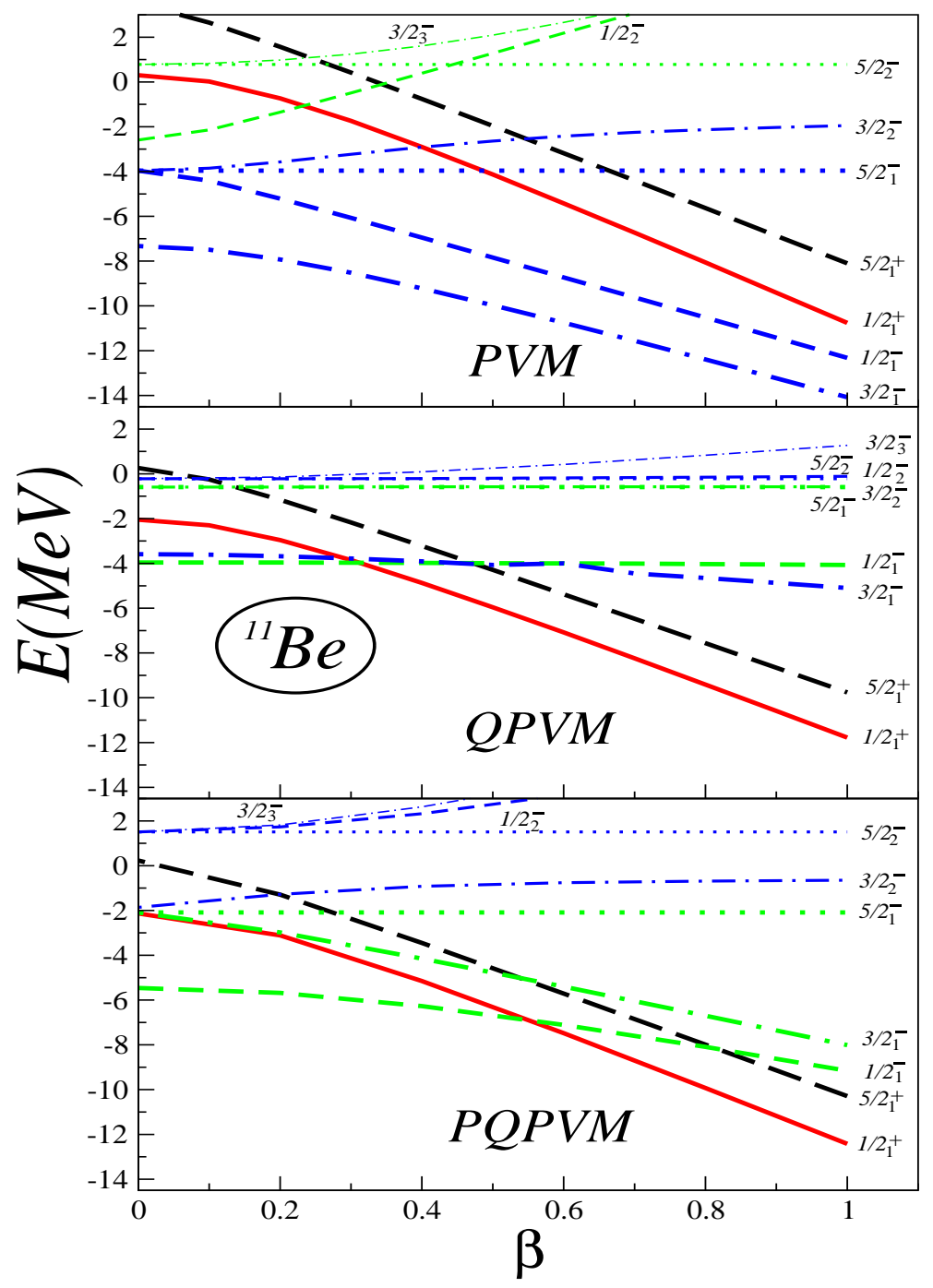

FIG. 7: (Color online) Calculated levels for ${ }^{11} \mathrm{Be}$ as functions of the $\beta$ value for ${ }^{10} \mathrm{Be}$, within the PVM, QPVM and PQPVM models.

$1 / 2_{1}^{-}$and $3 / 2_{1}^{-}$. Once more, as seen from the PQPVM results (lower panel), this is achieved through the number projection procedure only.

Both the $1 / 2_{1}^{+}$and $1 / 2_{1}^{-}$levels go down within the PQPVM when $\beta$ is increased, and their crossing happens close to the experimental value for the deformation parameter $(\beta \cong 0.6)$. Thus, the spin inversion mechanism is quite different here than in the PRM and PVM cases, where the $1 / 2_{1}^{-}$state is pushed up while the $1 / 2_{1}^{+}$state is pushed down $[3,9]$. The fact that in the PQPVM the $1 / 2_{1}^{-}$state is almost independent on the value of $\beta$ while the $1 / 2_{1}^{+}$state 


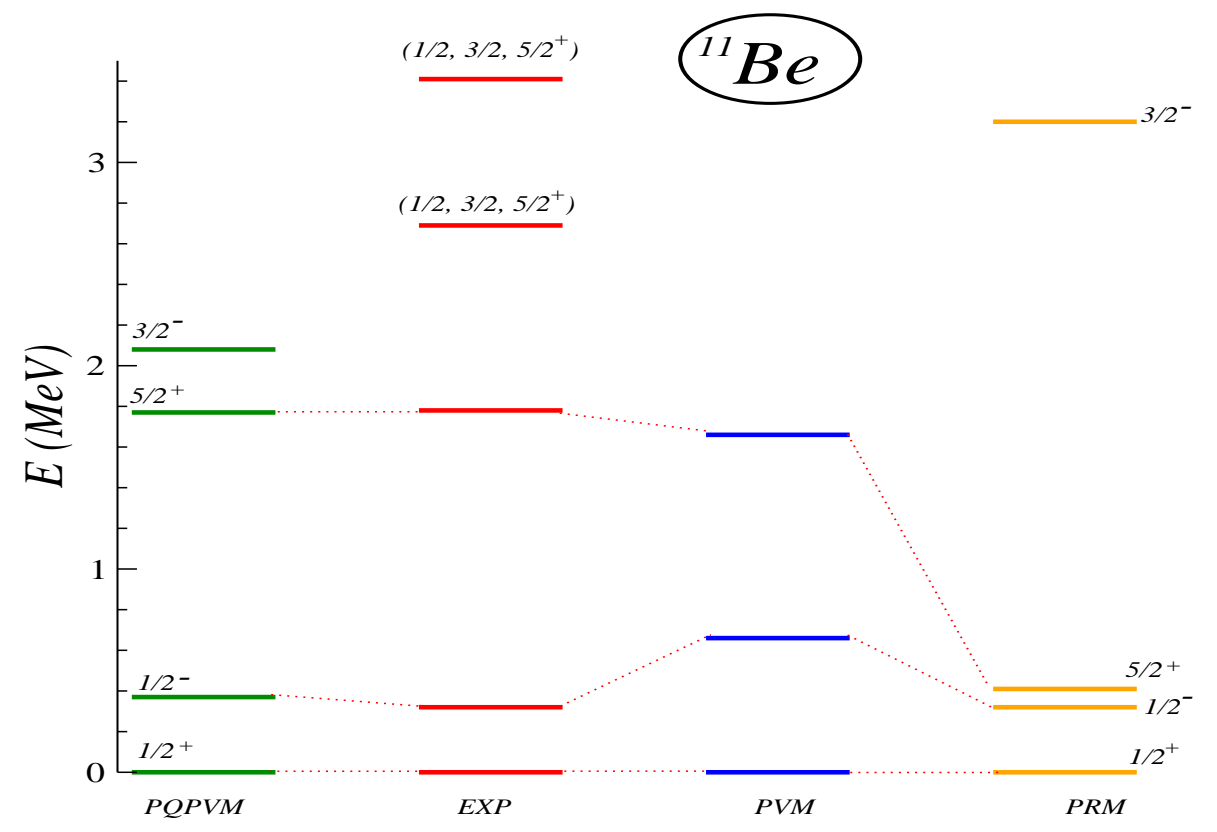

FIG. 8: (Color online) The low-energy spectra in ${ }^{11}$ Be within the PQPVM for $\beta=0.6$, compared with the experimental levels (EXP) and the PVM [3] and PRM [9] calculations.

varies very strongly is due to the $\mathrm{PP}$ factor given in the equation (3.3), which is small in the first case $\left(u_{p_{1 / 2}} \cong v_{p_{1 / 2}}\right)$ and large in second case $\left(u_{p_{1 / 2}} \cong 1, v_{p_{1 / 2}} \cong 0\right)$. Therefore, it can be argued that within the particle-core model the spin inversion in ${ }^{11} \mathrm{Be}$ is due to the $\mathrm{PP}$.

As shown in Fig. 8 all three lowest states in ${ }^{11} \mathrm{Be}$ are satisfactorily reproduced within the PQPVM for $\beta=0.6$. For the sake of comparison in the same figure are also presented the results of the PVM [3] and PRM [9] calculations.

The resulting PVM, QPVM and PQPVM wave functions for the lowest $1 / 2^{-}, 3 / 2^{-}, 1 / 2^{+}$, and $5 / 2^{+}$states in ${ }^{11} \mathrm{~B}$, with $\beta=0.6$, are confronted with previous calculations $[3,7,9,61]$ in Table V. All said above in relation of omission of levels based on configurations $\left|1 p_{3 / 2}, 0^{+}\right\rangle$ and $\left|1 p_{3 / 2}, 2^{+}\right\rangle$, and the percentages of the wave function amplitudes in the case of ${ }^{13} \mathrm{C}$ is valid here.

The PVM wave function for the $1 / 2_{1}^{-}$state, obtained by Vinh Mau [3], is quite similar to the one we get within the same model. Notice that the pairing tends to make this state 
TABLE V: Comparison between the wave functions and the spectroscopic factors obtained in the present work for the lowest states in ${ }^{11}$ Be with those derived by Vinh Mau [3] within the PVM, and by Nunes et al [9] and Esbensen et al [7] within the PRM, the variation shell model (VSM) of Otsuka et al [61]. and the shell model study done by Cohen and Kurath [19]. In all our calculations (PVM, QPVM, PQPVM) we adopt $\beta=0.60$.

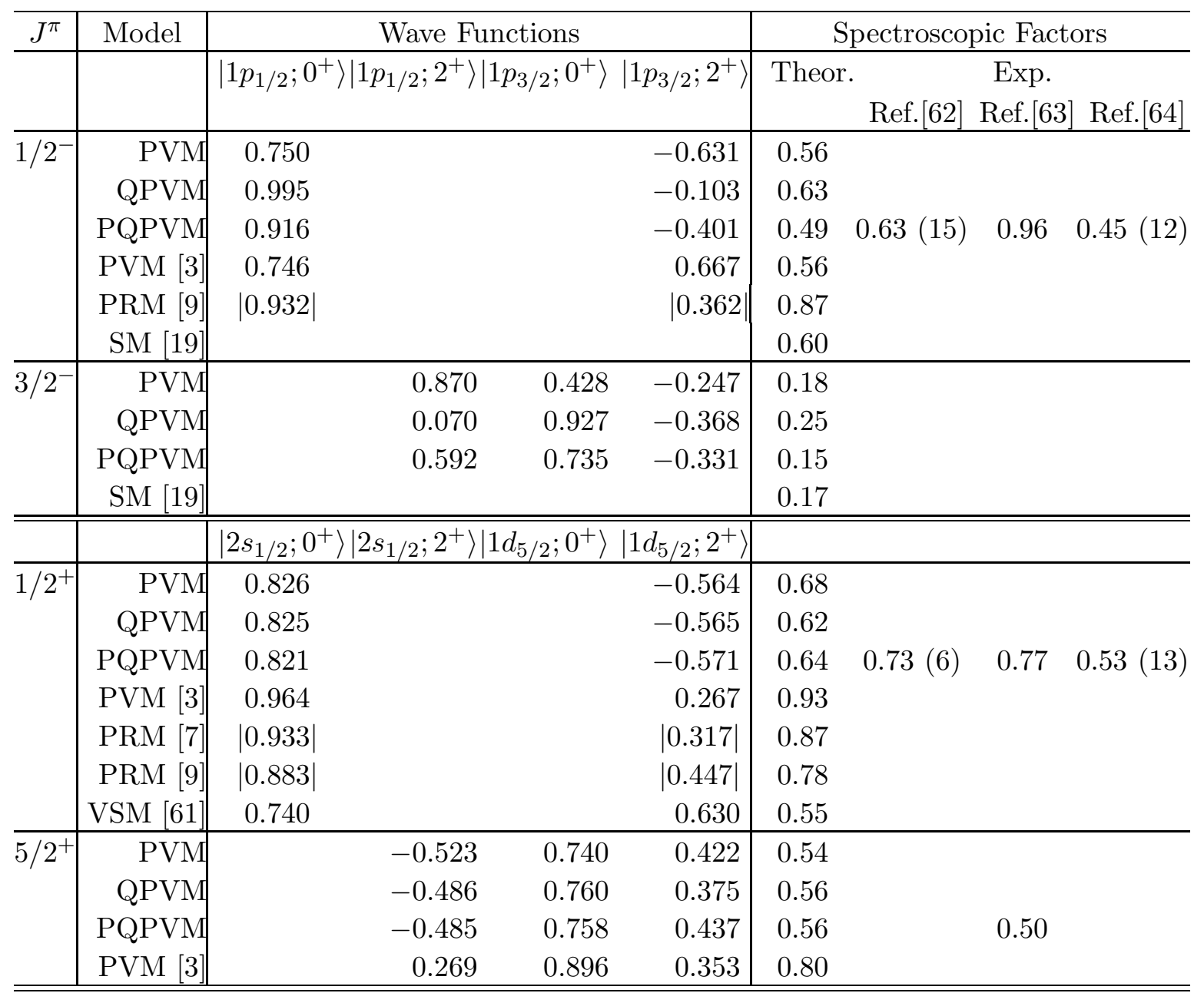

to be less collective. The effect is still more pronounced for the $3 / 2_{1}^{-}$state.

All three models yields similar results for the positive parity wave functions. When confronted with that derived within the PVM by Vinh Mau [3], one sees that, at variance with what happens with negative parity states, our wave functions are more collective. We also note that our wave function for the $1 / 2_{1}^{+}$state is similar to that obtained in the variational shell model (VSM) calculation of Otsuka et al Ref. [61].

The reaction spectroscopic factors for pickup on the ${ }^{11}$ Be target and for stripping on the 
${ }^{10} \mathrm{Be}$ target, evaluated with the wave functions listed in Table $\mathrm{V}$ are presented in the same table. We can read the differences with the previous PRM results for the $1 / 2_{1}^{+}$state $[7,9]$, and the similarity with the shell model calculation for the negative parity states [19].It can be observed that the experimental data [62-64] for the levels $1 / 2_{1}^{+}, 1 / 2_{1}^{-}$and $5 / 2_{1}^{+}$are reproduced quite well by the PQPVM.

Before ending this section, and in order to inquire on how realistic the above particle-core wave functions are, we briefly discuss the ground state magnetic dipoles moments in ${ }^{13} \mathrm{C}$ and ${ }^{11}$ Be. After some Racah algebra and by denoting $a$ and $b$, respectively, the single-particle and collective components in the wave functions, we get:

$$
\begin{aligned}
\mu\left(1 / 2_{1}^{-}\right) & =a^{2} \mu\left(1 p_{1 / 2}\right)-\frac{b^{2}}{3} \mu\left(1 p_{3 / 2}\right)+\frac{b^{2}}{2} \mu\left(2^{+}\right) \\
& =-\frac{g_{s}}{6}+b^{2} g_{R}
\end{aligned}
$$

and

$$
\begin{aligned}
\mu\left(1 / 2_{1}^{+}\right) & =a^{2} \mu\left(2 s_{1 / 2}\right)+\frac{7 b^{2}}{15} \mu\left(1 d_{5 / 2}\right)-\frac{b^{2}}{3} \mu\left(2^{+}\right) \\
& =\frac{g_{s}}{2}\left(a^{2}+\frac{7 b^{2}}{15}\right)-\frac{2 b^{2}}{3} g_{R},
\end{aligned}
$$

where $g_{s}=-3.82$ and $g_{R}=Z / A$ are, respectively, the effective spin and collective gyromagnetic ratios $[48,55]$.

TABLE VI: Magnetic dipole moments of the ground states in ${ }^{13} \mathrm{C}\left(1 / 2_{1}^{-}\right)$and ${ }^{11} \mathrm{Be}\left(1 / 2_{1}^{+}\right)$evaluated

\begin{tabular}{|c|c|c|c|c|c|c|}
\hline \multirow[t]{2}{*}{ Nucleus } & \multicolumn{5}{|c|}{ Theory } & Experiment \\
\hline & PCM QPCM & PQPCM & Ref.[3] & Ref.[9] Ref.[7] & Ref.[61] & Ref. [65] \\
\hline${ }^{13} \mathrm{C}$ & $0.77 \quad 0.63$ & 0.67 & 0.80 & 0.94 & - & $0.7024118(14)$ \\
\hline${ }^{11} \mathrm{Be}$ & $-1.66-1.67$ & -1.66 & -1.85 & $-1.78-1.71$ & -1.49 & $-1.6816(8)$ \\
\hline
\end{tabular}
with the wave functions listed in Tables IV and V are confronted with the experimental data. PCM stands here for the particle-core model, with the core being a rotor in the case of ${ }^{13} \mathrm{C}$, and a vibrator in the case of ${ }^{11} \mathrm{Be}$, etc.

The numerical results are shown in Table VI, from where one can see that they the magnetic dipole moments strongly depend on the interplay between the single-particle and collective degrees of freedom. 


\section{SUMMARY AND CONCLUSIONS}

Our work was divided into two main stages. First, we apply pure BCS and PBCS models to describe heavy carbon isotopes. We adopt the single-particle energies and pairing strengths which reproduce simultaneously the experimental binding energies of ${ }^{11} \mathrm{C}$ and ${ }^{13} \mathrm{C}$, and the low-energy spectra of ${ }^{13} \mathrm{C}$, except for the $3 / 2_{1}^{-}$state, which is nicely reproduced within the PBCS but not within the BCS (see Fig. 2). With these parameters we next evaluate the low-lying states in ${ }^{15} \mathrm{C},{ }^{17} \mathrm{C}$ and ${ }^{19} \mathrm{C}$. We found that both models are capable to explain fairly well the decrease of the binding energies in going from ${ }^{13} \mathrm{C}$ to ${ }^{19} \mathrm{C}$. The PBCS model reproduces as well the experimentally observed spin ordering $1 / 2^{+}, 5 / 2^{+}, 3 / 2^{+}$ of the lowest three states and the energy of the first $3 / 2^{-}$state in ${ }^{15} \mathrm{C}$. In addition, the same model correctly predicts the lowest $1 / 2^{+}$and $5 / 2^{+}$levels in ${ }^{17} \mathrm{C}$ and ${ }^{19} \mathrm{C}$, but it is not able to account for the $3 / 2_{1}^{+}$level in these two nuclei, which is very likely build up mainly on the anomalous seniority-three state $\left|\left(1 d_{5 / 2}\right)^{3} J^{\pi}=3 / 2^{+}\right\rangle$.

We have calculated the one-particle transfer spectroscopic factors for the lowest $1 / 2^{-}, 3 / 2^{-}, 1 / 2^{+}$and $5 / 2^{+}$states for all four odd-mass $\mathrm{C}$ isotopes obtaining quite similar results within the two pairing models. In ${ }^{13} \mathrm{C}$ the calculations agree with the experimental data for the ground state, but they fail to reproduce the experimental spectroscopic factors for excited states in this nucleus. For the heavier carbon isotopes the agreement with the experimental data is better. This implies that the low-lying states in ${ }^{15,17,19} \mathrm{C}$ are basically seniority-one states, while the contribution of seniority-three states and the collective effects could be important in ${ }^{13} \mathrm{C}$. Our results clearly indicate that the pairing interaction plays a major role in the nuclear structure of heavy carbon isotopes, partly accounting for their basic feature such as: a) small binding energies, b) spin-parity ordering of the low-lying states, and c) systematic decrease in the binding (of the order of $5 \mathrm{MeV}$ ) when one goes from ${ }^{13} \mathrm{C}$ to ${ }^{19} \mathrm{C}$. All this is achieved without employing any free parameter. Therefore, the pairing has to be taken into account in any theoretical calculation that aspire to be quantitatively realistic.

In the following stage we included the collective degrees of freedom in the framework of the weak-coupling model and applied it to describe ${ }^{13} \mathrm{C}$ and ${ }^{11} \mathrm{Be}$. Our main objective here was to analyze how the short range pairing correlations modify the core-particle coupling mechanism, and consequently the energy spectra, spectroscopic factors, and the magnetic 
dipole moments in these nuclei. As far as we know such a study has not been done so far, at least not in a systematic way ${ }^{6}$. It is worthwhile to stress once more that the PP, which is usually omitted in the simple particle-rotor and particle-vibrator coupling models, is brought up back into the play by the inclusion of the pairing. Here the single-particle energies were taken from the standard parametrization for the Wood-Saxon potential, while the coupling matrix elements were calculated using the wave functions of the harmonic oscillator. The last procedure could be a rather crude approach for a weakly bound nucleus such as ${ }^{11}$ Be. However, we feel that it is, nevertheless, good enough to reveal the importance of the PP

We found that only PQPRM reproduces satisfactorily the experimental ordering of the lowest four levels in ${ }^{13} \mathrm{C}$ with the correct value of deformation $\beta=-0.6$. Neither the PRM nor the QPRM could accomplish this. The pairing strongly reduces the coupling between the $0^{+}$and the $2^{+}$core states for the negative parity states $1 / 2^{-}$and $3 / 2^{-}$. As a consequence, within the QPRM and PQPRM the ground state of ${ }^{13} \mathrm{C}$ turns out to be basically the singleparticle $1 p_{1 / 2}$ state. Our positive parity states $1 / 2^{+}$and $5 / 2^{+}$, on the other hand, are more collective when compared to results of the previous works. Both effects seems to go in right direction and make the PQRPM to account for the experimental spectroscopic factors of low lying positive and negative parity states, and for the dipole magnetic moment of the ground state.

Similar calculations were done for one-neutron halo nucleus ${ }^{11} \mathrm{Be}$. It is found that the more likely structure of the core, ${ }^{10} \mathrm{Be}$, required to reproduce the lowest three states in ${ }^{11} \mathrm{Be}$, is that of a vibrator. Moreover, we feel that the appropriate model for the experimental value of the vibrational length, $\beta=0.6$, is again the PQPVM. As before, we found that inclusion of pairing makes the negative-parity states less collective and positive parity states more collective compared to earlier works. Also here the spectroscopic factors and the dipole magnetic moment are well reproduced by the PQPVM.

In summary, the inclusion of the pairing interaction and of the concomitant Pauli Principle is imperative not only in the case of heavy odd-mass carbon isotopes but also in the core-coupling models of ${ }^{13} \mathrm{C}$ and ${ }^{11} \mathrm{Be}$. The important role played by the particle number conservation in these relatively light and/or exotic nuclei has been confirmed as well.

\footnotetext{
${ }^{6}$ See however the Refs. $[5,6,18]$.
} 


\section{Acknowledgments}

Authors would like to acknowledge the partial support of Brazilian agencies Conselho Nacional de Ciência e Tecnologia (CNPq) and Fundação de Amparo à Pesquisa do Estado

de São Paulo (FAPESP). A.R.S. acknowledges support received from Fundação de Amparo à Pesquisa do Estado do Rio de Janeiro (FAPERJ).

[1] M. Bender, P.H. Heenen, and P.G Reinhard, Rev. Mod. Phys. 75, 121 (2003).

[2] J. Dobaczewski, and W. Nazarewicz, Prog. Theor. Phys. Suppl. 146, 70 (2003).

[3] N. Vinh Mau, Nucl. Phys. A592, 33 (1995).

[4] G. Coló, Toshio Suzuki, and H. Sagawa, Nucl. Phys. A695, 167 (2001).

[5] G. Gori, F. Barranco, E. Vigezzi, and R. A. Broglia, Phys. Rev. C 69, 041302(R) (2004).

[6] R.A. Broglia, F. Barranco, G. Coló, G. Gori, E. Vigezzi, and P.F. Bortignon, Eur. Phys. J. A 20, 81 (2004).

[7] H. Esbensen, B.A. Brown, and H. Sagawa, Phys. Rev. C 51, 1274 (1995).

[8] F.M. Nunes, Core Excitation in Few Body Systems: Application to halo nuclei, (PhD thesis, University of Surrey, 1995, unpublished).

[9] F.M. Nunes, I.J. Thompson, and R.C. Johnson, Nucl. Phys. A596, 171 (1996).

[10] D. Ridikas, M.H. Smedberg, J.S. Vaagen and M.V. Zhukov, Nucl. Phys. A628, 363 (1998).

[11] I. Talmi and I. Unna, Phys. Rev. Lett 4, 469 (1960).

[12] C. Forssén, P. Navrátil, W. E. Ormand, and E. Caurier, Phys. Rev. C 71, 044312 (2005).

[13] H. Sagawa, B.A. Brown, and H. Esbensen, Phys. Lett. B309, 1 (1993).

[14] A.M. Lane, Proc. Phys. Soc. (London) A68, 1978 (1955).

[15] D. Kurath and R.D. Lawson, Nucl. Phys. 23, 5 (1961).

[16] O. Mikoshiba, T. Terasawa, and M. Tanifuji, Nucl. Phys. A168, 417 (1971).

[17] A. Robson and W.J. Van Megen, Nucl. Phys. A184, 67 (1972).

[18] F. Barranco, P.F. Bortignon, R.A. Broglia, G. Coló, and E. Vigezzi, Eur. Phys. J. A 11, 385 (2001).

[19] S. Cohen and D. Kurath, Nuc. Phys. A101, 1 (1967).

[20] T. Suzuki, R. Fujimoto and T. Otsuka, Phys. Rev. C 67, 044302 (2003). 
[21] P.L. Ottaviani, and M. Savoia, Phys. Rev. 178, 1594 (1969); Phys. Rev. 187, 1306 (1969), Nuovo Cim. 67A, 653 (1970).

[22] F. Krmpotić, A. Mariano, T.T.S. Kuo, and K. Nakayama Phys. Lett. B319, 393 (1993).

[23] F. Krmpotić, A. Mariano, and A. Samana, Phys. Lett. B541, 298 (2002).

[24] F. Krmpotić, A. Mariano, and A. Samana, Phys. Rev. C 71, 44319 (2005).

[25] L. S. Kisslinger and R. A. Sorensen, Rev. Mod. Phys. 35, 853 (1963).

[26] W. Scholz and F.B. Malik, Phys. Rev. 176, 1355 (1968).

[27] K.W.C. Steward, B. Castel, and B.P. Singh, Phys. Rev. C 4, 2131 (1971).

[28] B. Barman Roy, and D.C. Choudhury, Phys. Rev. C 12, 323 (1975).

[29] B.L. Cohen and R.E. Price, Phys. Rev. 121, 1441 (1960).

[30] V. Gillet and N. Vinh Mau, Nuc. Phys. 54, 321 (1964).

[31] P.J. Siemens and A.S. Jensen, Elements of Nuclei: Many Body Physics with the Strong Interction (Addison-Wesley Publishing Company Inc., Redwood City, California, 1987).

[32] F. Ajzenberg-Selove, Nucl. Phys. A523, 1 (1991), TUNL Nuclear Data Evaluation Project, available WWW: http://www.tunl.duke.edu/nucldata/, National Nuclear Data Center, Brookhaven National Laboratory, webpage http://www.nndc.bnl.gov/nudat2/.

[33] G. Murillo, S. Sen, and S.E. Darden, Nucl. Phys. A579, 125 (1994).

[34] H. G. Bohlen, R. Kalpakchieva, B. Gebauer, S. M. Grimes, H. Lenske, K. P. Lieb, T. N. Massey, M. Milin, W. von Oertzen, Ch. Schulz, T. Kokalova, S. Torilov, and S. Thummerer, Phys. Rev. C 68054606 (2003).

[35] F. Cappuzzello, S. E. A. Orrigo, A. Cunsolo, H. Lenske, M. C. Allia, D. Beaumel, S. Fortier, A. Foti, A. Lazzaro, C. Nociforo and J. S. Winfield, Europhys. Lett. 65, 766 (2004).

[36] Z. Elekes, Z. Dombradi, R. Kanungo, H. Baba, Z. Fulop, J. Gibelin, A. Horvath, E. Ideguchi, Y. Ichikawa, N. Iwasa, H. Iwasaki, S. Kanno, S. Kawai, Y. Kondo, T. Motobayashi, M. Notani, T. Ohnishi, A. Ozawa, H. Sakurai, S. Shimoura, E. Takeshita, S. Takeuchi, I. Tanihata, Y. Togano, C. Wu, Y. Yamaguchi, Y. Yanagisawa, A. Yoshida, and K.Yoshida, Phys. Lett. B614, 174 (2005).

[37] H. G. Bohlen, R. Kalpakchieva, W. von Oertzen, T. N. Massey, A.A. Ogloblin, G. de Angelis, Ch. Schulz, T. Kokalova, and C. Wheldon, J. Phys. G 31, S1461 (2005).

[38] M.B. Tsang, Jenny Lee, and W.G. Lynch, http://arXiv.org/abs/nucl-ex/0506016.

[39] V. Maddalena, T. Aumann, D. Bazin, B. A. Brown, J. A. Caggiano, B. Davids, T. Glasmacher, 
P. G. Hansen, R. W. Ibbotson, A. Navin, B. V. Pritychenko, H. Scheit, B. M. Sherrill, M. Steiner, J. A. Tostevin, and J. Yurkon, Phys.Rev. C 63, 024613 (2001).

[40] B. A. Brown, Prog. Part. Nucl. Phys 47, 517 (2001).

[41] D. Bazin, W. Benenson, B.A. Brown, J. Brown, B. Davids, M. Fauerbach, P.G. Hansen, P. Mantica, D.J. Morrissey, C.F. Powell, B.M. Sherrill, and M. Steiner, Phys. Rev. C 57, 2156 (1998).

[42] X.D. Liu, M.A. Famiano, W.G. Lynch, M. B. Tsang, and J. A. Tostevin, Phys. Rev. C 69, $064313(2004)$.

[43] H. Ohnuma, N. Hoshino, O. Mikoshiba, K. Raywood, A. Sakaguchi, G.G. Shute, B.M. Spicer, M.H. Tanaka, M. Tanifuji, T. Terasawa, and M. Yasue, Nuc. Phys. A448, 205 (1985).

[44] S. Truog and H.T. Fortuna, Phys. Rev. C 28977 (1983).

[45] G. Thiamova, N. Itagaki, T. Otsuka, and K. Ikeda, Eur. Phys. J. A22, 461 (2004).

[46] D.R. Tilley, H.R. Weller and C.M. Cheves, Nucl. Phys. A564, 1 (1993).

[47] E.K. Warburton and B.A. Brown, Phys. Rev. C 46, 923 (1992).

[48] A. Bohr and B.R. Mottelson, Nuclear Structure, vol.II, (W.A. Benjamin, Inc. 1975).

[49] D. Kurath, Phys.Rev. 80, 98 (1950).

[50] D. Bazin, B. A. Brown, J. Brown, M. Fauerbach, M. Hellström, S. E. Hirzebruch, J. H. Kelley, R. A. Kryger, D. J. Morrissey, R. Pfaff, C. F. Powell, B. M. Sherrill, and M. Thoennessen, Phys. Rev. Lett 74, 3569 (1995).

[51] T. Nakamura, N. Fukuda, T. Kobayashi, N. Aoi, H. Iwasaki, T. Kubo, A. Mengoni, M. Notani, H. Otsu, H. Sakurai, S. Shimoura, T. Teranishi, Y. X. Watanabe, K. Yoneda, and M. Ishihara, Phys. Rev. Lett. 83, 1112 (1999).

[52] D. Cortina-Gil, T. Baumann, H. Geissel, H. Lenske, K. Sümmerer, L. Axelsson, U. Bergmann, M.J.G. Borge, L.M. Fraile, M. Hellström, M. Ivanov, N. Iwasa, R. Janik, B. Jonson, K. Markenroth, G. Münzenberg, F. Nickel, T. Nilsson, A. Ozawa, K. Riisager, G. Schrieder, W. Schwab, H. Simon, C. Scheidenberger, B. Sitar, T. Suzuki and M. Winkler, Eur. Phys. J. A10, 49 (2001).

[53] G. Audi and A.H. Wapstra, Nucl. Phys. A565, 1 (1993); G. Audi, O Besillon, J. Blashot and A.H. Wapstra, ibid. A624, 1 (1997).

[54] G. Audi, A.H. Wapstra and C. Thibault, Nucl. Phys. A729, 337 (2003).

[55] A. Bohr and B.R. Mottelson, Nuclear Structure, vol. I, (W.A. Benjamin, Inc. 1969). 
[56] W.J. Vermeer, M.T. Esat, J.A. Kuehner, R.H. Spear, A.M. Baxter and S. Hinds, Phys. Lett. B122, 23 (1983).

[57] C.A. Pearson, J.M. Covan, D. Zissermann, T.G. Miller, F.P. Gibson, R. Haglund, W. Morrison and G. Westley, Nuc. Phys. A191, 1 (1972).

[58] D. J. Millener, D. I. Sober, H. Crannell, J. T. O’Brien, W. Fagg, S. Kowalski, C. F. Williamson, and L. Lapiks, Phys. Rev. C 39, 14 (1989).

[59] H. U. Jager, H. R. Kissener, and R. A. Eramzhian, Nuc. Phys. A171, 16 (1971).

[60] H. Iwasaki, T. Motobayashi, H. Akiyoshi, Y. Ando, N. Fukuda, H. Fujiwara, Zs. Flp, K. I. Hahn, Y. Higurashi, M. Hirai, I. Hisanaga, N. Iwasa, T. Kijima, T. Minemura, T. Nakamura, M. Notani, S. Ozawa, H. Sakurai, S. Shimoura, S. Takeuchi, T. Teranishi, Y. Yanagisawa and M. Ishiharaa, Phys. Lett. B481, 7 (2000).

[61] T. Otsuka, N. Fukunishi and H. Sagawa, Phys. Rev. Lett. 70, 1385 (1993).

[62] D.L. Auton, Nuc. Phys. A157, 305 (1970).

[63] B. Zwieglinsky, W. Benenson and R.G.H. Robertson, Nuc. Phys. A315, 124 (1979).

[64] A. Navin, D.W. Anthony, T. Aumann, T. Baumann, D. Bazin, Y. Blumenfeld, B.A. Brown, T. Glasmacher, P. G. Hansen, R.W. Ibbotson, P.A. Lofy, V. Maddalena, K. Miller, T. Nakamura, B. V. Pritychenko, B.M. Sherrill, E. Spears, M. Steiner, J.A. Tostevin, J. Yurkon, and A. Wagner, Phys. Rev. Lett. 85, 266 (2000).

[65] N.J. Stone, Atomic Data and Nuclear Data Tables 90, 75 (2005). 